\title{
BMJ Open Second opinion programmes in Germany: a mixed-methods study protocol
}

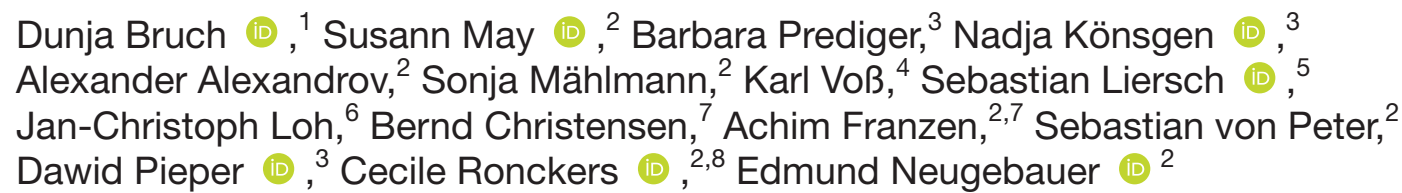

To cite: Bruch D, May S, Prediger B, et al. Second opinion programmes in Germany: a mixed-methods study protocol. BMJ Open 2021;11:e045264. doi:10.1136/ bmjopen-2020-045264

- Prepublication history for this paper is available online. To view these files, please visit the journal online (http://dx.doi org/10.1136/bmjopen-2020045264).

CR and EN are joint senior authors.

Received 25 September 2020 Revised 29 December 2020 Accepted 19 January 2021

Check for updates

(C) Author(s) (or their employer(s)) 2021. Re-use permitted under CC BY-NC. No commercial re-use. See rights and permissions. Published by BMJ.

For numbered affiliations see end of article.

Correspondence to

Dunja Bruch;

dunja.bruch@mhb-fontane.de

\section{ABSTRACT}

Introduction Second opinion programmes aim to support the patients' decision-making process and to avoid treatments that are unnecessary from a medical perspective. The German second opinion directive, introduced in December 2018, constitutes a new legal framework in statutory health insurance for seeking second opinions for elective procedures and so far includes tonsillectomy, tonsillotomy, hysterectomy and shoulder arthroscopy. The directive mandates physicians who recommend one of the above-mentioned surgeries to inform their patients of their legal right to visit a certified second opinion provider. Since second opinion programmes are a fairly recent phenomenon in Germany, no comprehensive data are yet available on the degree of implementation, users, potential barriers and their effectiveness. We aim to examine the characteristics and the use of second opinion programmes as well as the needs and wishes from the perspective of (potential) users in Germany, with focus on the decision-making process, the patient-physician relationship and the motivation to seek a second opinion, as well as the role of health literacy.

Methods and analysis Six substudies will include the following stakeholders: (1 and 2) patients with one of the four surgery-indications covered by the directive, (3) patients who electively sought an online-based second opinion, (4) patients with oncological diseases, (5) the general population and (6) medical specialists. A mixedmethods approach will be used, including questionnaires, interviews and focus groups. The data will be evaluated using quantitative descriptive analysis and qualitative content analysis. The integration of the results will take place in the form of a triangulation protocol.

Ethics and dissemination The study protocol was approved by the Ethics Committee of the Brandenburg Medical School. The findings will be published in peerreviewed journals and presented at scientific conferences.

\section{INTRODUCTION}

Surgical second opinion programmes are known worldwide since the 1970 s. ${ }^{12}$ Historically, these programmes were introduced in the USA to halt the increasing numbers of surgeries, as a means of limiting
Strengths and limitations of this study

- We address the perspectives of various important stakeholders such as patients and physicians in order to obtain a comprehensive overview of the use of and the need for a second medical opinion.

- Because of the mixed-methods approach, we are able to provide real-life contextual understandings and multilevel perspectives.

- The mixed-methods design allows to reconsider the research question for each substudy and to develop questionnaires and interview guidelines iteratively.

- Since we survey very specific patient groups in some substudies, we are dependent on close cooperation with eligible patients and physicians in order to reach the target number of participants.

rising healthcare costs. ${ }^{1}$ Second opinion programmes offer patients with a recommendation for an elective surgical procedure the opportunity to obtain a second opinion from another medical professional. The primary aims of these programmes are to provide an improved knowledge base, to support the patient in the decision-making process and to avoid treatments that are unnecessary from a medical perspective.

In Germany, many statutory health insurers offer a wide variety of second opinion programmes with an increasing volume. ${ }^{3}$ Moreover, it is fairly common among patients to use an informal approach to obtain a second opinion, by seeking medical advice from multiple healthcare practitioners for the same set of symptoms and diagnostic test results, before the patient makes a final decision on proposed interventions. This 'informal' patient-initiated second opinion practice is tolerated by the statutory health insurance and usually reimbursed without clear regulations. ${ }^{4}$ The second opinion 
programmes on the other hand are structured and subject to clear regulations.

In December 2018, the Federal Joint Committee published the second opinion directive, ${ }^{5}$ which introduced the first statutory second opinion programme covered by the German statutory health insurance for a specified set of elective surgeries: Initially, the directive included only tonsillectomy, tonsillotomy and hysterectomy. In 2020, shoulder arthroscopy was added. A widening of the selected surgeries in the directive is anticipated in the future. A physician, who recommends one of the selected surgeries to patients who are members of the statutory health insurance, is obliged to inform the patient about his/her right to obtain a second opinion. The physician must also provide further information, for example, where the patient can find second-opinion physicians and decision aids. Of note, surgical interventions in oncology are explicitly excluded from the directive in its current form and online-based second opinions are not supposed to be provided yet according to the second opinion directive.

As structural second opinion programmes are a fairly recent phenomenon in Germany, there have not been any comprehensive efforts to gather data on the degree of implementation, users, potential barriers, acceptance and benefits of the second opinion programmes, for example, value for informed decision making or reduction of surgeries, except for a few small-scale evaluations. ${ }^{6}$ As such, the scientific basis for the design of second opinion programmes in general, and the criteria for selection of the relevant (surgical) indications is limited.

Studies show that there is a noticeable interest among German citizens to seek a second opinion. ${ }^{78}$ Moreover, a population representative study ${ }^{7}$ shows that the need for a second opinion goes beyond the indications included in the second opinion directive: more than half of the respondents consider the possibility of a second opinion to be important not only for surgeries involving bones and joints $(56 \%)$, and for surgeries on internal organs $(56 \%)$, but also for other types of medical interventions, such as drug treatments in case of cancer $(70 \%)$ and for radiotherapy $(61 \%)$. Although generally speaking, health information is increasingly sought online, $90 \%$ of the study participants prefer personal contact with a specialist when they seek a second opinion. Only $10 \%$ preferred medical advice by phone or online. ${ }^{7}$

The ZWEIT Project Needs analysis and review of current practice regarding second opinion in medicine (original: Bestandsaufnahme und Bedarfsanalyse von medizinischen ZWEITmeinungsverfahren in Deutschland) was set up by the Brandenburg Medical School and the Witten/Herdecke University in cooperation with the Association of Statutory Health Insurance Physicians Brandenburg, the statutory health insurer AOK Nordost and an online-based second opinion provider, Medexo.

\section{Objectives}

The study is particularly important right now because the second opinion directive recently created a new legal framework for second opinion programmes. This has also an impact on existing second opinion programmes in health insurance. For example, health insurers offer online second opinion programmes only as long as they do not include the surgeries specified by the directive. As the second opinion directive can replace existing second opinion programmes offered by health insurers, it is important that the effects of the directive will be evaluated to what extent they meet the needs of patients and how feasible the directive is for physicians.

The objective of the ZWEIT Project is to examine the characteristics and the use of second opinion programmes as well as the needs and wishes from the perspective of (potential) users and physicians. By revealing and summarising the experiences and the needs of stakeholders in the healthcare system, we aim to provide decision-makers in health policy with important information to support further tailoring of second opinion programmes.

\section{METHODS AND ANALYSIS}

\section{Rationale for the mixed-methods approach}

The mixed-methods approach combines the strengths of qualitative and quantitative research and is appropriate for research questions that require real-life contextual understandings and multilevel perspectives. ${ }^{9}$ Our questionnaires aim to interrogate a broad spectrum of (potential) patient populations to obtain a comprehensive and representative overview of the need for and the use of second opinions. Questionnaire-based research is rigid and provides a high potential for comparability across populations. The qualitative investigations enable to formulate additional questions and items for the questionnaires, attending to aspects that have previously not been considered. ${ }^{10}$ Further, they allow an in-depth analysis of the subjective experiences and attitudes of the study participants. ${ }^{11}$ Thereby, the results of the questionnaires can be deepened and contextualised by using expert and problem-centred interviews, ${ }^{12}$ as well as focus groups. ${ }^{13}$ Owing to this methodological complexity, the research questions to be addressed expand beyond the a priori hypotheses developed by the research team. ${ }^{11}$

\section{Design}

The study follows a multiphase design. ${ }^{9}$ Quantitative and qualitative methods will be performed in parallel, except for group 2, for which we chose a sequential order. ${ }^{14}$ Figure 1 shows a summary of the study design, methods and specific objectives.

As the focus is on the perspective of the user, we will include specific patient groups and physicians. In groups 1 and 2, we will concentrate on patients whose indications are within the scope of the second opinion directive. We will compare patients with the indication given before 


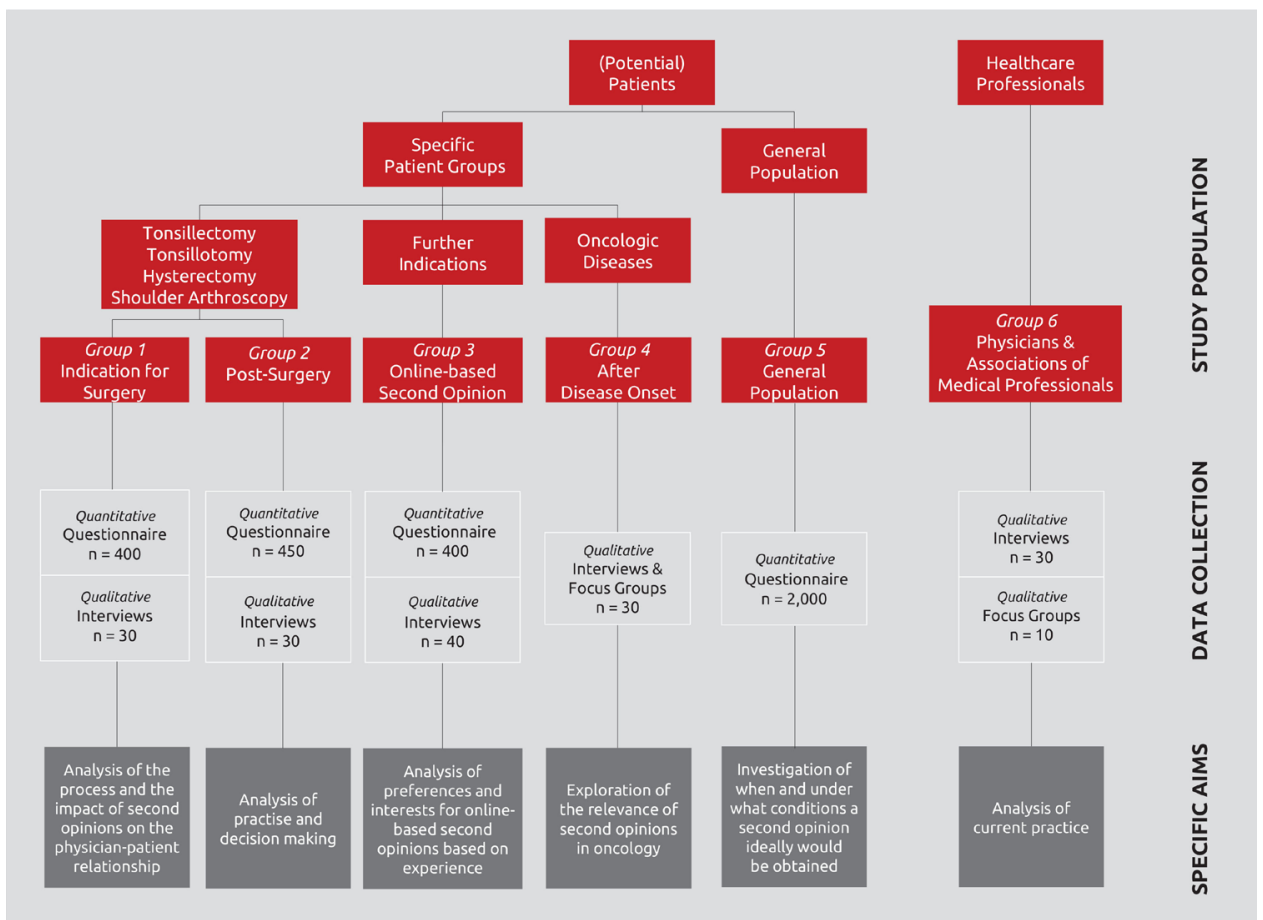

Figure 1 Bestandsaufnahme und Bedarfsanalyse von medizinischen ZWEITmeinungsverfahren in Deutschland (Needs analysis and review of current practice regarding second opinion in medicine) - (ZWEIT) project study design.

and after the introduction of the directive. In group 3, we will survey patients who have obtained a second opinion via an online platform. Online-based second opinion programmes are offered by several health insurers. Since the second opinion directive excludes so far online-based second opinions, these programmes can only be provided by statutory health insurers for surgeries that are not part of the directive. At the same time, online-based second opinions could compensate for regional differences in healthcare, such as a lack of medical specialists. The second opinion directive does not apply to oncological diseases. However, it is known from preliminary studies that there is a need for a second opinion in oncology ${ }^{7}$ and that many statutory health insurers offer second opinion programmes for oncological indications. ${ }^{3}$ We will continue to explore the need for a second oncological opinion in group 4 so that future second opinion programmes can be tailored to the characteristics of specific oncological patient populations. In addition, we will perform a representative survey of the general population in order to analyse the needs in the general population, for example, with regard to the relevant indications. In order to investigate the experience with the second opinion directive and the requirements for a successful second opinion process from a professional point of view, we will interview physicians.

\section{Group 1: patients who received indication for surgery} Specific aims

We will focus on the analysis of the second opinion process and the impact of the second opinion directive on the patient-physician relationship.

\section{Study population}

This group will include people who have received an indication for tonsillectomy, tonsillotomy, hysterectomy or shoulder arthroscopy. Further inclusion criteria will be: insured by the statutory health insurance, sufficient knowledge of the German language and age $\geq 18$ or parent and legal guardian, respectively, willing to complete the questionnaire.

We will recruit physicians specialised in otolaryngology, gynaecology and orthopaedics in the federal states of Berlin and Brandenburg, based on registries from the Association of Statutory Health Insurance Physicians Brandenburg. We will focus on outpatient settings because these physicians commonly provide the surgical indication for the abovementioned procedures. In the next step, the physicians will recruit participants by distributing the questionnaires and invitations for interviews to their patients who meet the inclusion criteria. Participating physicians will receive a remuneration of $€ 5$ for each invited patient (regardless of whether the patient participates or not). According to the total number of tonsillectomies, tonsillotomies and hysterectomies (approx. $157120^{15}$ ) in relation to the total number of outpatient gynaecologists and ENT physicians (approx. $16024^{16}$ ) in Germany in 2017, we assume an average of 20 eligible patients per physician within a data collection period of 2 years. With 65 cooperating physicians we assume 1300 eligible patients. With a response rate of $30 \%,{ }^{17}$ we expect a sample size of $n=400$.

\section{Outcomes of interest}

We will focus on the implementation of the second opinion directive and the quality of information the physician provides to the patient, for example, information 
about which physicians provide a second opinion and where to find decision aids. We will investigate whether a second opinion was required, what reasons exist for or against obtaining a second opinion and what sort of wishes patients have with regard to a second opinion programme, for example, preference for a personal or online-based second opinion or quality demands on the medical specialist. Furthermore, we will analyse the impact of the directive on the patient-physician relationship as well as the effect of health literacy on obtaining a second opinion.

\section{Group 2: patients who have undergone tonsillectomy, tonsillotomy, hysterectomy or shoulder arthroscopy Specific aims}

We will evaluate the use of second opinions as well as their relevance for the decision behaviour in patients who already decided on undergoing the surgeries specified by the second opinion directive.

\section{Study population}

We will include patients who have undergone tonsillectomy, tonsillotomy, hysterectomy or shoulder arthroscopy due to non-malignant underlying diseases in the period 2018-2019. Initially, a preliminary qualitative interview study will include eligible adults (age $>18$ years) to identify main themes of interest. We will recruit those from clinics and outpatient physicians. Subsequently, the full study will recruit individuals who are insured by the AOK Nordost and who are either adults or, in the case of minors, whose parents or legal guardians are willing to complete the questionnaire. AOK Nordost is a German health insurer in the federal states of Berlin, Brandenburg and Mecklenburg-Western Pomerania covering approximately 1,76 million insured citizens. Eligible individuals will receive a postal invitation from AOK Nordost to participate in the questionnaire survey and the interviews. Patients who underwent surgery before the second opinion directive was introduced will receive the same questionnaire with specific additional items. AOK Nordost will send a postcard reminder after 2 weeks. Based on the case numbers from previous years (approx. 9000 per year) and response rates (5\%-10\%) based on the experience of the AOK Nordost from similar surveys, the estimated number of responses is at least 450 in a conservative scenario. As the second opinion directive on these indications was enacted in December 2018, this study allows a comparison of patients who had surgery before and after the onset of the directive.

\section{Outcomes of interest}

This part of the study will focus on the patient's informed decision-making process and identify knowledge and needs related to obtaining a second opinion, as well as factors that influence the use. Furthermore, we will compare the impact of the second opinion directives on receiving a second opinion between patients who have undergone surgery before and after the introduction of the directive. In addition, a model of the decision typology of people who have had surgery will be generated. We will also analyse the impact of health literacy on obtaining a second opinion.

\section{Group 3: patients who obtained an online-based second opinion \\ Specific aims}

Our aim will be to survey patient experiences with an online-based second opinion programme.

\section{Study population}

We will include clients of an online platform (Medexo). Medexo provides a written, medical record based second opinion to patients.

Medexo will send out study invitation to all its customers in Germany (1247) from January 2016 to February 2019. Non-responders will receive up to two reminders Assuming a conventional response rate for postal surveys of $30 \%,{ }^{17}$ we plan to recruit 400 participants. Accordingly, a heterogeneous sample will be chosen for the interviews in which the characteristics of the individuals differ as much as possible.

\section{Outcomes of interest}

This part of the project will focus on the motivation to obtain an online-based second opinion and the experiences made during the second opinion process. The investigation includes potential discrepancies between first and second opinion, the impact of the second opinion on the participants' decision and on the patientphysician relationship. In addition, we will analyse health literacy and the perceived advantages and disadvantages of an online-based second opinion.

\section{Group 4: patients with oncological diseases \\ Specific aims}

We will explore the relevance of second opinion programmes in oncology.

\section{Study population}

We will include members of oncological support groups with a sample size of up to 30 subjects for problem-centred interviews or focus groups. Advanced cases and palliative situations will be excluded. We will identify eligible organisations through online research and contacts with individual oncologists and patient representatives.

\section{Outcomes of interest}

This part of the study explores whether patients with cancer have previously obtained a second opinion or whether there was a need for it.

\section{Group 5: General population}

Specific aims

We aim to analyse the use of second opinions as well as the needs in the general population and identify possible structural and regional differences. 


\section{Study population}

We based the sample size $n=2000$ on the last representative survey on the subject of second opinion in Germany by Geraedts and $\mathrm{Kraska}^{7}$ and also considered an evenly distributed number of cases for rural, urban and agglomeration areas. Assuming a response rate of $20 \%$, we will select a random sample of 9990 citizens living in the region of Berlin and the state of Brandenburg aged 18 years or older through regional registration offices. We will select a random sample of 9990 citizens living in the region of Berlin and the state of Brandenburg aged 18 years or older through regional registration offices. For selection of participants, we will use disproportionate stratified sampling with settlement pattern (urban area, area with agglomeration, rural area) as stratification variable. For each settlement pattern, we will contact the same number of citizens. We will randomly select all 5 municipalities in urban areas, 10 in areas with agglomeration and 10 in rural areas. The five urban municipalities will each select 666 persons of their data randomly. The 20 other municipalities will each select 333 persons of their data randomly. We will send questionnaires to the whole sample with the opportunity to win one of 125 gift cards for Amazon ( $€ 50$ each) as an incentive. Six weeks later, we will send a reminder.

\section{Outcomes of interest}

This part of the study will focus on knowledge, attitudes and wishes towards a second opinion programme in the context of general health literacy and the local care situation. In this context, we will also analyse the impact of health literacy on obtaining a second opinion.

\section{Group 6: Specialists and professional medical associations Specific aims}

We will focus on the process of and attitudes towards second opinions as well as the influence of the second opinion directive on daily practise.

\section{Study population}

Eligible practitioners will consist of specialists affected by the second opinion directive. In addition, we will conduct expert interviews with representatives of professional associations. Thirty specialists and 10 representatives from professional associations will be included.

The sample will consist of already cooperating specialists from group 1 and additionally selected physicians. The sample is balanced between physicians who work in rural and urban regions, as well as whether the physicians offer second opinions or not.

\section{Outcomes of interest}

We will examine subjective perspectives on second opinions as well as the implementation of the second opinion directive by medical specialists.

\section{Data collection}

We will collect the data via questionnaires, interviews and focus groups (table 1 ).
Table 1 Instruments, data collection and data analysis for study groups 1-6

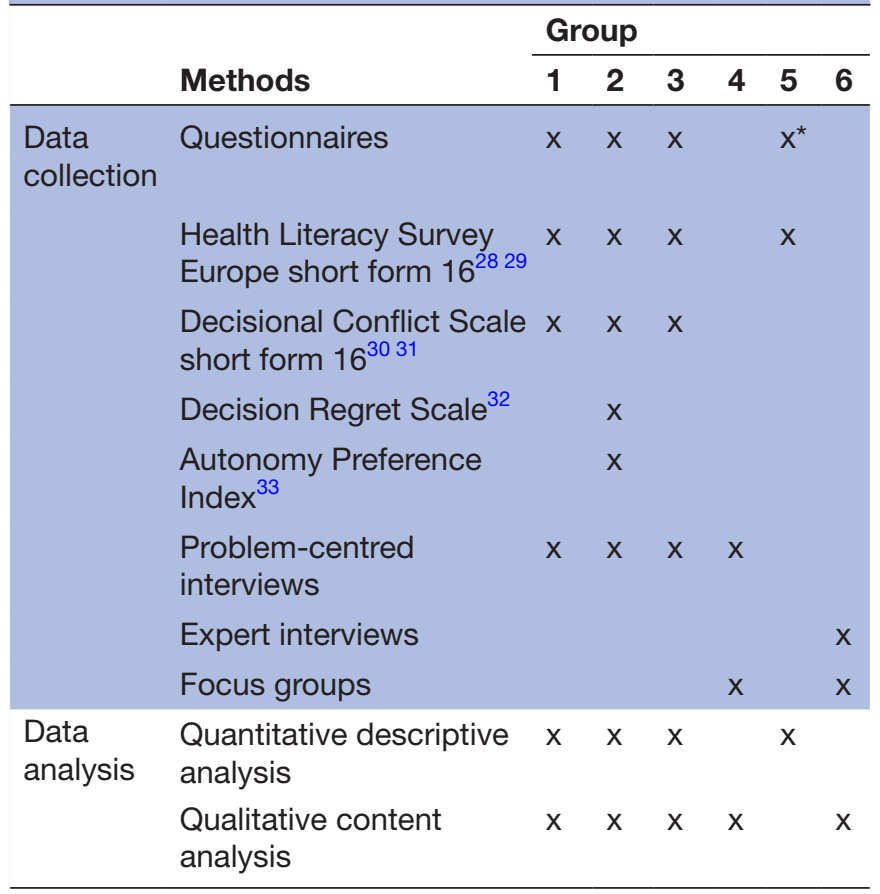

Group 1, patients who received indication for surgery of tonsillectomy, tonsillotomy, hysterectomy or shoulder arthroscopy; 2, patients who have undergone tonsillectomy, tonsillotomy, hysterectomy or shoulder arthroscopy; 3 , patients who obtained an online-based second opinion;4, patients with oncological diseases; 5 , general population; 6 , specialists and professional medical associations.

*Some questions will follow a survey conducted in Germany by Geraedts and Kraska. ${ }^{7}$

\section{Questionnaires}

We will develop the respective questionnaires in several interdisciplinary meetings. All study questionnaires will be piloted in person or via phone among patients who match the inclusion criteria of each group to ensure good comprehensibility and to optimise the reading flow.

The core of each group-specific questionnaire will consist of validated instruments to match the specific aims and outcomes of the respective substudies, as shown in table 1. We will develop additional items specifically for the substudies focusing on experiences and wishes towards second opinions, decisional behaviour and local healthcare characteristics. Health-related and sociodemographic data will also be included where appropriate in the substudies: disease, duration of symptoms and severity, decision preferences, age, gender, marital status, educational level, income and rural versus urban residence.

The holders of the patient data who collect and store information in conjunction with their main duties (eg, the AOK Nordost or Medexo) will sent out questionnaires for groups 2 and 3. Returning the postal questionnaire to the University Study Team, who have no access 
to personal identifiers of the invited individuals, will be free of charge for the participants. We will use paper-andpencil questionnaires except for group 2, where we will provide additional online surveys. Since it is optional for the patients to participate in the survey, we will examine sampling bias by comparing the characteristics of the respondents to the non-respondents, for example, where feasible by age and gender.

\section{Interviews and focus groups}

For the patient study, an interview guide will be developed for undertaking problem-centred interviews ${ }^{12} 18$ and focus groups. ${ }^{13} \mathrm{~A}$ short questionnaire will be added to collect basic data and personal characteristics. ${ }^{18}$ In addition, an interview guide for the expert interviews with physicians will be constructed based on a literature search and the results of the patient interviews. The interview protocol contains a section of questions about experiencing the second opinion process, if applicable. Furthermore, the participants are asked about their preferences regarding the development of second opinion offers. Each substudy contains a topicspecific block: group 1: impact of the second opinion on the patient-physician relationship; group 2: decisionmaking behaviour; group 3: lack of personal contact due to an online-based second opinion; group 4: social conditions; group 6: impact on daily practice. Expert and problem-centred interviews will be conducted in person or by phone. The interview sample will be selected according to the maximum variation criterion. ${ }^{19}$ Relevant criteria are age, gender and residence (rural vs urban). All interviews and focus groups will be transcribed word for word. ${ }^{20}$

\section{Data analysis}

We will use quantitative descriptive analysis for the questionnaires and qualitative content analysis for the interviews and focus groups (table 1 ).

\section{Quantitative descriptive analysis}

We will focus on descriptive analyses of patient demographics and reported outcomes to characterise the dataset. Where feasible, we will examine associations of sociodemographic or health-related factors such as age, gender, health literacy, population density of residence, decisional conflict and education for each substudy. Based on this and on univariate analysis, we might consider a stepwise regression analysis to be performed.

\section{Qualitative content analysis}

The structured qualitative content analysis by Kuckartz ${ }^{21}$ enables a rule-driven reduction and systematisation of the data. In the first phase, we will create categories describing the material. This procedure is inductive and is carried out on the transcript with regard to the question. The aim is to develop an exhaustive category system. The first phase will be performed in parallel for data collection. In the second phase, we will apply the developed category system to the entire material. For the second phase, the data collection must already be completed. To ensure traceability, we will validate the application of the category system by a member check. ${ }^{22} 23$

\section{Integration of the data}

First, the responses to the questions that are unspecific to the involved interventions will be compared descriptively between the substudies, such as the participants' preferences towards the type of the second opinion (online-based second opinion on the basis of documents versus personally provided second opinion) or whether they have previously obtained a second opinion. Second, the quantitative and qualitative results will be integrated using a triangulation protocol. ${ }^{24}$ This means a separated analysis of data and their subsequent presentation side by side in a single document. ${ }^{1424}$ This technique allows to consider where there is agreement, partial agreement, silence or dissonance between findings from different methods. ${ }^{24}$ Furthermore, the qualitative data will be used to deepen the quantitative findings, and thus, to enable their more complex understanding, ${ }^{14}$ for example, the reasons for especially positive or negative attitudes may be understood in more detail or for rejecting of a second opinion programme.

\section{DISCUSSION}

The second opinion directive has created a systematic offer to obtain second opinions for certain surgical procedures in Germany. The present study aims to examine the use of second opinions prior to and since the introduction of the second opinion directive. Additionally, we will elucidate the benefits and drawbacks of the newly introduced second opinion framework in the context of the associated surgical procedures. Moreover, we will investigate topics that are not yet included in the directive, such as online-based second opinion programmes and second opinions regarding cancer treatments. ${ }^{5}$ In parallel, the project team updated knowledge on the current state of affairs regarding second opinion programmes by surveying all health insurers (statutory and private) about their programmes. These programmes continue to be offered in addition to the second opinion directive. The results of our study, together with the results of the survey among health insurers, will provide useful information and further guidance for decision-makers to implement more tailored second opinion programmes and to stimulate specific future research addressing the knowledge gaps identified in our endeavour.

The study explores the extent to which individuals require health literacy as a competence for obtaining a second opinion. ${ }^{25}$ In addition, health literacy is dependent on structural factors and thus represents a societal responsibility. ${ }^{26}$ Accordingly, organisations that provide health services must provide barrier-free access to healthrelated and healthcare-related information. Second opinion programmes aim to support the patient in 
making informed decisions. The ZWEIT Project provides empirical evidence the extent to which the current second opinion framework offers the patient valid and complete information for making informed decisions, and, if desired, for obtaining a second opinion.

Our study has various strengths: on the microlevel and mesolevel, we address opinions, wishes, experiences and needs of various important stakeholders, including clinicians of a variety of disciplines, patients who are presurgery and postsurgery, clients of a commercial second opinion provider and the general population. The questionnaires will be tailored for each of the stakeholders, yet will also contain selected overlapping items. As such, a comparison of the different stakeholders' views and experiences will be possible across the full spectrum of stakeholders, in particular for the selected validated item scales. The survey on postsurgery patients (group 2) will include patients pre and post the commencement of the second opinion directive. We aim to check if and how the directive was implemented in daily clinical practice. The survey on the general population will consider the local care situation specifically and how second opinion programmes are feasible in rural areas.

Limitations should be noted as well. To a certain extent, physicians who have a positive attitude towards second opinions may be more likely to participate in the study and to educate their patients about the right to obtain a second opinion than physicians with a negative attitude. This can lead to a bias in the sample of patients recruited through physicians (group 1).

In the second opinion directive, the inclusion of other professional groups such as physiotherapists or psychologists is not prohibited, but is neither encouraged. Assuming that a second opinion on shoulder arthroscopy may lead to a decrease in surgeries, more patients may receive conservative treatment including physiotherapy. ${ }^{27}$ In further research, other medical professions should also be considered, such as physiotherapist. In addition, the perspective of other stakeholders (such as legal experts and policy-makers) should also be taken into account in order to analyse the feasibility and requirements of the directive at the macro level.

\section{ETHICS AND DISSEMINATION}

The study protocol was approved by the Ethics Committee of the Brandenburg Medical School on 13 June 2019 (number E-01-20190529). All quantitative research will use data anonymisation procedures compliant with the General Data Protection Regulation. For the interviews and focus groups, the participants are asked to sign an informed consent form. The transcription of the audiotapes and the analysis will be undertaken using pseudonymisation. The participants in the focus groups and interviews are offered an allowance.

The findings will be published in peer-reviewed journals and presented at scientific conferences. In addition, a symposium will be planned at the end of the project,
Table 2 (Expected) dates for the completion of data collection and analysis in group 1-6 and status for data collection at the time of the first revision

\begin{tabular}{|c|c|c|c|}
\hline Group & $\begin{array}{l}\text { Data collection } \\
\text { completed }\end{array}$ & $\begin{array}{l}\text { Status data } \\
\text { collection }\end{array}$ & $\begin{array}{l}\text { Data analysis } \\
\text { completed }\end{array}$ \\
\hline 1 & 31 July 2021 & Ongoing & 31 October 2021 \\
\hline 2 & 30 June 2021 & Ongoing & $\begin{array}{l}30 \text { September } \\
2021\end{array}$ \\
\hline 3 & 31 January 2020 & Completed & 31 March 2021 \\
\hline 4 & 30 June 2021 & Not yet initiated & 31 August 2021 \\
\hline 5 & 31 October 2020 & Completed & 30 June 2021 \\
\hline 6 & 30 April 2021 & Ongoing & 31 June 2021 \\
\hline
\end{tabular}

to enable a wide range of stakeholders to take note of the results, and to provide their professional opinion and critical assessment of the conclusions and the potential impact on the second opinion directive.

\section{DATA STATEMENT}

The datasets generated during the study are not currently publicly available due to the study being ongoing. Data will be available from the corresponding author on reasonable request once the study is completed. Data generated or analysed during the study will be included in manuscripts to be submitted for publication in peerreviewed journals.

\section{Patient and public involvement statement}

The patients were not involved in the design of the study. However, a patient representative is member of the Scientific Advisory Board of the ZWEIT project.

\section{Study status}

In table 2, we show the study status for each substudy and dates for (expected) completion of data collection and analysis.

\section{Author affiliations}

${ }^{1}$ Faculty of Health Sciences Brandenburg, Brandenburg Medical School Theodor Fontane, Neuruppin, Germany

${ }^{2}$ Brandenburg Medical School Theodor Fontane, Neuruppin, Germany

${ }^{3}$ Institute for Research in Operative Medicine, University Witten Herdecke Faculty of Health, Cologne, Germany

${ }^{4}$ Association of Statutory Health Insurance Physicians Brandenburg, Potsdam, Germany

${ }^{5}$ AOK Nordost, Strategic healthcare analytics/GeWINO, Berlin, Germany

${ }^{6}$ Medexo GmbH, Berlin, Germany

${ }^{7}$ Ruppin clinics, Neuruppin, Germany

${ }^{8}$ Princess Maxima Center for Pediatric Oncology, Utrecht, The Netherlands

Acknowledgements The ZWEIT Study Group acknowledges the contributions of the Scientific Advisory Board (Nicole Ernstmann, Martin Härter, Gerd Glaeske, Ilona Köster-Steinebach, Dominik von Stillfried, Monika Nothacker). Kristin Schnuppe (Coordinating Centre for Clinical Studies, Medical University Brandenburg) provided support with the data protection concept.

Contributors DB, CR, SuM, BP and NK drafted the manuscript. EN, DP, SvP, $\mathrm{KV}, \mathrm{J}-\mathrm{CL}$ and $\mathrm{DB}$ are coapplicants on the funded grant and contributed to conceptualisation of the study design. AA, SoM, AF, BC and SL are members of the 
study team that have contributed to specify the study design. All coauthors have revised the manuscript critically for important intellectual content.

Funding This work was supported by the Innovation Committee at the Federal Joint Committee (Innovation Fund, grant number 01VSF18014).

Competing interests DB, SuM, BP, NK, AA, SoM, KV, SL, BC, AF, SvP, DP, CR and EN declare that they have no competing interests. J-CL is the CEO of Medexo, a second opinion provider.

Patient and public involvement Patients and/or the public were not involved in the design, or conduct, or reporting, or dissemination plans of this research.

Patient consent for publication Not required.

Provenance and peer review Not commissioned; externally peer reviewed.

Open access This is an open access article distributed in accordance with the Creative Commons Attribution Non Commercial (CC BY-NC 4.0) license, which permits others to distribute, remix, adapt, build upon this work non-commercially, and license their derivative works on different terms, provided the original work is properly cited, appropriate credit is given, any changes made indicated, and the use is non-commercial. See: http://creativecommons.org/licenses/by-nc/4.0/.

\section{ORCID iDs}

Dunja Bruch http://orcid.org/0000-0002-9511-8487

Susann May http://orcid.org/0000-0003-3847-4861

Nadja Könsgen http://orcid.org/0000-0002-5387-7119

Sebastian Liersch http://orcid.org/0000-0002-8613-4434

Dawid Pieper http://orcid.org/0000-0002-0715-5182

Cecile Ronckers http://orcid.org/0000-0003-3524-4657

Edmund Neugebauer http://orcid.org/0000-0002-6192-8692

\section{REFERENCES}

1 Lindsey PA, Newhouse JP. The cost and value of second surgical opinion programs: a critical review of the literature. J Health Polit Policy Law 1990;15:543-70.

2 Grafe WR, McSherry CK, Finkel ML, et al. The elective surgery second opinion program. Ann Surg 1978;188:323-30.

3 Pieper D, Heß S, Mathes T. Bestandsaufnahme zu Zweitmeinungsverfahren in Der Gesetzlichen Krankenversicherung (GKV). Gesundheitswesen 2018;80:859-63.

4 GKV-Spitzenverband. Stellungnahme zum Referentenentwurf des GKV-Versorgungsstärkungsgesetzes. Available: file:///D:/Literatur\%20ZWEIT/Stellungnahmen/GKVVersorgungsstärkungsgesetz/GKV-SV_zu_GKV-VSG.pdf

5 Gemeinsamer Bundesausschuss. Richtlinie zum Zweitmeinungsverfahren. Bundesanzeiger, 2020.

6 Ali J, Pieper D. Kaum aktuelle Daten zu Zweitmeinungsverfahren vorhanden - eine systematische Übersichtsarbeit. Gesundheitswesen 2017;79:871-4.

7 Geraedts M, Kraska R. Zweitmeinungen: Inanspruchnahme und Bedarf AUS Sicht Der Bevölkerung. Gesundheitsmonitor 2016. Bertelsmann Stiftung, 2016: 160-77.

8 Weyerstraß J, Prediger B, Neugebauer E, et al. [First results of a German second opinion program show high patient satisfaction and large discrepancies between initial therapy recommendations and second opinion]. Z Evid Fortbild Qual Gesundhwes 2018;133:46-50.

9 Creswell JW, Klassen AC, Plano Clark VL. Best practices for mixed methods research in the health sciences. Bethesda: National Institutes of Health, 2013: 541-5.

10 Craig P, Dieppe P, Macintyre S, et al. Developing and evaluating complex interventions: the new medical Research Council guidance. BMJ 2008;337:a1655.
11 Flick U, Ev K, Steinke I. Was ist qualitative Forschung? Einleitung und Überblick. In: Flick U, Ev K, Steinke I, eds. Qualitative Forschung: ein Handbuch. 11th edn. Reinbek: Rowohlt Taschenbuch, 2015: 13-29.

12 Mey G, Mruck K. Interviews. In: Mey G, Mruck K, eds. Handbuch qualitative Forschung in Der Psychologie. Wiesbaden: Springer, 2010: 423-35.

13 Krueger RA. Focus groups: a practical guide for applied research. Sage publications, 2014

14 Creswell JW, Hirose M. Mixed methods and survey research in family medicine and community health. Fam Med Community Health 2019;7:e000086.

15 Statistisches Bundesamt. Fallpauschalenbezogene Krankenhausstatistik, 2017. Available: https://www.destatis.de

16 Bundesärztekammer. Ärztestatistik zum. Available: https://www. bundesaerztekammer.de/fileadmin/user_upload/downloads/pdfOrdner/Statistik2017/Stat17AbbTab.pdf [Accessed 31. Dec 2017].

17 Clearinghouse for Military Family Readiness. Survey response rates: rapid literature review. University Park: Clearinghouse for Military Family Readiness, 2019.

18 Witzel A. Das problemzentrierte interview. Beltz, 1985.

19 Patton MQ. Qualitative research \& evaluation methods: Integrating theory and practice. Sage publications, 2014.

20 Dresing T, Pehl T. Praxisbuch interview, transkription \& analyse anleitungen und regelsysteme für qualitativ Forschende. Marburg: Dr. Dresing \& Pehl GmbH, 2012.

21 Kuckartz U. Qualitative Inhaltsanalyse. 4th ed. Weinheim: Beltz Juventa, 2018.

22 Kuper A, Lingard L, Levinson W. Critically appraising qualitative research. BMJ 2008;337:a1035.

23 Onwuegbuzie AJ, Leech NL. Validity and qualitative research: an oxymoron? Qual Quant 2007;41:233-49.

24 O'Cathain A, Murphy E, Nicholl J. Three techniques for integrating data in mixed methods studies. BMJ 2010;341:c4587.

25 Sørensen K, Van den Broucke S, Fullam J, et al. Health literacy and public health: a systematic review and integration of definitions and models. BMC Public Health 2012;12:80.

26 Ernstmann N, Bauer U, Berens E-M. DNVF memorandum Gesundheitskompetenz (Teil 1) - Hintergrund, Relevanz, Gegenstand und Fragestellungen in Der Versorgungsforschung. Das Gesundheitswesen 2020;82:e77-93.

27 Steuri R, Sattelmayer M, Elsig S, et al. Effectiveness of conservative interventions including exercise, manual therapy and medical management in adults with shoulder impingement: a systematic review and meta-analysis of RCTs. Br J Sports Med 2017;51:1340-7.

28 Pelikan JM, Ganahl K. Die europäische GesundheitskompetenzStudie: Konzept, instrument und ausgewählte Ergebnisse. health literacy, Forschungsstand und Perspektiven. 1st ed. Bern: Hogrefe, 2017: 93-126.

29 Pelikan JM, Ganahl K. Measuring health literacy in general populations: primary findings from the HLS-EU Consortium's health literacy assessment effort. Stud Health Technol Inform 2017;240:34-59.

30 Buchholz A, Hölzel L, Kriston L, et al. Die decisional conflict scale in deutscher sprache (DCS-D)-dimensionale struktur in einer stichprobe von hausarztpatienten. Klinische Diagnostik und Evaluation 2011;4:15-30.

31 O'Connor AM. Validation of a decisional conflict scale. Med Decis Making 1995;15:25-30.

32 Brehaut JC, O'Connor AM, Wood TJ, et al. Validation of a decision regret scale. Med Decis Making 2003;23:281-92.

33 Simon D, Kriston L, Härter M. Die deutsche modifizierte Fassung des Autonomie-Präferenz-Index (API-Dm). Klinische Diagnostik und Evaluation 2011;4:5-14 